\title{
PRESENTACIÓN: \\ CAMPESINOS, COMERCIANTES Y FUNCIONARIOS EN LA HISTORIA DE LAS SOCIEDADES LATINOAMERICANAS: EL LEGADO DE JUAN CARLOS GARAVAGLIA (1944-2017)
}

\section{Peasants, traders, and civil servants in the history of Latín American societies: the legacy of Juan Carlos Garavaglia (1944-2017)}

Que una enfermedad implacable pusiese fin a la vida de Juan Carlos Garavaglia en aquel enero de 2017 resultó algo muy difícil de asimilar para sus amigos y colegas. Tantos proyectos inconclusos y tantos planes personales, emprendidos siempre con un raro entusiasmo vital, constituyeron para muchos que lo tratamos con asiduidad parte de nuestras vidas, algo que uno termina considerando normal, habitual; algo que es así, porque así son las cosas que se dan absurdamente por supuestas.

Entre este grupo de sorprendidos observadores de Juan figuramos los colegas que en la Universidad Pompeu Fabra de Barcelona recibimos como un factor de crecimiento intelectual de primer orden la incorporación de Juan en la década de 1990. No por casualidad, el primer artículo publicado en Illes i Imperis fue ya en el año 2001. Esta relación continuada en el quehacer habitual del grupo de historiadores que terminaron confluyendo en la Universitat Pompeu Fabra fue el resultado de una triple conjunción de factores. El primero que debe considerarse es la larga relación profesional y personal establecida con algunos de nosotros al calor de la renovación de los estudios en España y América colonial e independiente, de los nexos económicos e imperiales que unieron a los dos mundos. En este punto, fue probablemente una vieja relación de Juan con el Prof. Josep Fontana el nexo más antiguo, la pieza más remota de una relación que tuvo una continuidad cierta.

La segunda circunstancia o factor fue la formalización en una nueva universidad pública barcelonesa, la nuestra, fundada en los años noventa, de un núcleo que, auspiciado de nuevo por el Prof. Fontana, reemprendió algunos de los temas de interés transatlántico que antes ya habían propiciado reuniones realizadas en torno a ellos. Algunos ejemplos muestran aquella continuidad, cuestiones y problemas historiográficos que alumbraron el trabajo que se llevaría a cabo a partir de los años 1990s: historia de las sociedades coloniales y de la minería; condición de los pueblos nativos; impacto de las reformas borbónicas; crisis imperial y origen de las repúblicas independientes y de la España liberal (y colonial) del siglo XIX. No era un mal programa ni un programa corto de ambición, pero se trataba de un esquema de trabajo sólo concebible como el propio de una renova- 
ción historiográfica de toda la profesión dispuesta a cambiar los enfoques hasta donde fuese necesario, sin concesiones a los mitos de las historias nacionales y del etnocentrismo europeísta a la española (que ahora parece renacer) o del simétrico de los criollos herederos en las sociedades descolonizadas en 1810-1824.

El tercer factor es más evidente y fácil de explicitar: la concesión de un proyecto de investigación del European Research Council para investigar la formación del Estado moderno y contemporáneo en América Latina (con aportaciones para Europa y España). Para ello Garavaglia estableció su cuartel general en nuestra universidad, con la ayuda de la Prof. Claudia Contente. Para esta exigente singladura reclutó a un selecto grupo de jóvenes investigadores latinoamericanos y solicitó la ayuda de investigadores consolidados en aquellas otras historiografías con las que el historiador argentino aspiraba a comparar la experiencia americana. El trabajo de aquellos cinco años se reflejó puntualmente en trabajos publicados en distintos lugares y de modo fehaciente en los trabajos recogidos en los volúmenes State-Building, como Configuraciones estatales, regiones y sociedades locales. América Latina, siglos XIX-XX (Barcelona, 2011); Las Fuerzas de Guerra en la Construcción del Estado. América Latina siglo XIX (Rosario, 2012); Latin Amerian Bureaucracy and the State Building Process (1780-1860) (Newcastle upon Tyne, 2013); Serve the Power(s), Serve the State. America and Eurasia (Newcastle upon Tyne, 2016).

Los trabajos que se reúnen en el presente volumen son el resultado de la reunión celebrada en nuestra universidad para recordar a Juan Carlos. Insistimos en lo que ya se dijo. No es fácil aceptar psicológicamente la desaparición de una influencia intelectual tan poderosa, clave para la historiografía de su país y de los nuestros, puesto que cuando ambas historiografías se han separado el precio que se paga es alto coste. Como Juan sabía muy bien, la historia del mundo toma siempre forma entre unas gentes y unos paisajes concretos, tangibles. En efecto, si algo aprendimos del gran historiador que fue, se refiere a la importancia de identificar las cuestiones a tratar por su relevancia y profundidad, aquella cualidad que impide luego abandonarlas, a darlas jamás por concluidas. Por la relevancia y su continuada proyección sobre nuestras sociedades, todavía desiguales e injustas, las historias de campesinos, de comerciantes y de servidores del Estado primigenio, el legado de Juan Carlos Garavaglia sigue vivo entre nosotros.

Redacción de Illes i Imperis, octubre de 2018, julio de 2019 


\section{NOTA DE LA EDITORA}

Juan Carlos Garavaglia es el hilo conductor que une los trabajos que hemos reunido aquí. Si bien a primera vista podría parecer que el conjunto carece de coherencia, debemos advertir que no es éste el caso. Se trata de un compendio que ha sido cuidadosamente seleccionado por sus colegas y amigos en función de los temas que le interesaban. Son el resultado de charlas, de inquietudes compartidas, de pistas que contribuyó a explorar o que incentivó a algunos de nosotros a transitar. Son igualmente el resultado de las jornadas realizadas en nuestra universidad en octubre de 2018, y de la iniciativa de algunas personas que en ese momento no pudieron estar presentes. ${ }^{1}$ Todos ellos tenían proximidad con Juan Carlos Garavaglia y su obra.

Este dossier da una pauta - mínima, por cierto- de la diversidad de las cuestiones que le interesaban y que recorrieron su trayectoria. Por ejemplo, Víctor Hugo Acuña nos habla de la evolución de fiestas y conmemoraciones patrias costarricenses y observa como esos eventos, al mismo tiempo que encauzaban en nuevas direcciones sentimientos de pertenencia y memoria colectiva, se convirtieron paulatinamente en una forma de asumir una posición de subordinación con respecto al imperialismo estadounidense. Lo que conduce a otra cuestión, la de la relación entre memoria e historia y la posición del historiador ante esa memoria colectiva y representaciones del pasado, una cuestión que permite a Frédérique Langue apoyarse en la obra autobiográfica de Garavaglia, Una juventud en los años 60, para brindarnos una reflexión a propósito del compromiso político asumido por Juan Carlos, una instancia en la que se superpusieron los papeles de militante, testigo e historiador.

La construcción del Estado nación, la organización y funcionamiento de sus instituciones es otro de los temas que fascinó a Juan Carlos y que en 2008 le permitió obtener el Advanced Grant del European Research Council. Aquí se refleja en los textos de Mariana Canedo, Silvia Capanema y Mariana Flores. El primero de ellos se basa en las primeras elecciones municipales realizadas en la campaña del efímero Estado de Buenos Aires, para analizar las tensiones sociales que el proceso electoral puso de manifiesto en los pueblos. A través de estos eventos analiza como se articulan las discusiones en las Cámaras Legislativas con las prácticas electorales efectivamente constatadas en el mundo rural.

1. Parte de las comunicaciones de dichas jornadas serán publicadas en la obra compilada por Josep M. Fradera y Raúl Fradkin, Juan Carlos Garavaglia. La pasión por la historia, Buenos Aires, Prometeo Editorial (en prensa). 
En otros términos, las tensiones y desafíos entre poderes locales y poder central, entre sectores que se empeñan en legitimar, en consolidar sus posiciones. ${ }^{2}$

Los trabajos de Silvia Capanema y de Mariana Flores da Cunha Thompson Flores se centran en Brasil y tratan aspectos bien diferentes. Mientras el primero de ellos aborda el funcionamiento de la marina, los ámbitos en que se reclutaba a sus integrantes y en las decisiones tomadas en previsión de una guerra con sus vecinos — con la idea subyacente de una expansión imperial — en tiempos de la recién nacida República Brasileña, Mariana Flores da Cunha Thompson Flores nos traslada al espacio fronterizo sur del Imperio de Brasil en la primera mitad del siglo XIX. Su objetivo consiste en desvelar cómo se articularon diversas estrategias en la construcción del estado de esa zona periférica a través de la burocracia, el papel de las aduanas, de la fiscalidad, sin descuidar el ámbito de la justicia y su funcionamiento en relación con los diferentes grupos sociales.

Precisamente el estudio de zonas periféricas, que tal como nos lo recuerda María Elena Barral, fue uno de los ángulos de observación preferidos por Juan Carlos, es el adoptado también por Judith Farberman, que nos acerca a una zona tradicionalmente marginal, la provincia de Santiago del Estero. Lo hace a través de un aspecto prácticamente inexplorado al día de hoy, a saber, la tenencia de la tierra bajo la forma de campos colectivos y su evolución. Las relaciones sociales, vínculos de patronazgo, la división y explotación de estos campos, así como la especulación y pleitos de los que fueron objeto, son los ejes que articulan este trabajo. Dividir, medir la tierra, son nociones que, como es sabido, llevan implícito el control del territorio y Pierre Gautreau, con quien Juan Carlos abordó justamente el estudio de estas cuestiones en el mundo rural del siglo XIX rioplatense, ${ }^{3}$ nos aporta una derivación artística, y lúdica derivada de aquellas inquietudes.

El mundo rural con su gente anónima está igualmente presente en estas páginas. Lucila Bugallo nos introduce en el universo campesino de la Puna jujeña actual a través de sus prácticas, rituales, e incluso nostalgia por las «mingas», en vías de desaparición de un mundo que vive en profunda comunión con su medioambiente. M. Elena Barral analiza el recorrido historiográfico de Juan Carlos en relación a un aspecto bien preciso: el de las instituciones religiosas, la religiosidad y las creencias de la gente de a pie del espacio rioplatense. Pilar López Bejarano se ocupa de los sectores populares, los entramados sociales, la estratificación socio-racial y el mundo del trabajo en la Bogotá del siglo XVIII. Un trabajo de investigación cuyos primeros pasos fueron acompañados por el propio Juan Carlos. Finalmente, en la década de los noventa Garavaglia escribió un artículo con su inseparable amigo, Juan Carlos Grosso, acerca de los sectores populares, estratificación

2. Cabe destacar que el trabajo de M. Fernanda Barcos, «¿Qué hacer después de la guerra? Los prolegómenos del Estado de Buenos Aires», incluido en este mismo número de Illes i Imperis aporta valiosos elementos complementarios a propósito de las luchas, la búsqueda de consenso y legitimidad que enfrentó el Estado de Buenos Aires durante ese mismo periodo.

3. Garavaglia, Juan Carlos y Pierre Gautreau (comps.), Mensurar la tierra, controlar el territorio. América Latina, siglos XVIII-XIX, Rosario, Prohistoria Ediciones, 2011 
social y la noción de raza. Este artículo da pie al análisis de Alexandre Coello a propósito de la resonancia que este trabajo tuvo para la antropología y la historia y que, veinticinco años después de su publicación, sigue siendo de absoluta actualidad.

En fin, el lector encontrará aquí ecos de los diversos mundos por los que transitó Juan Carlos Garavaglia con su asumida condición de investigador obsesivo, sus cuestionamientos permanentes, con su pasión y entusiasmo irremplazables, que echamos en falta cada día.

Claudia Contente UPF/

GRIMSE

(Barcelona, España) 